\title{
Evaluación empírica de la calidad de la información: caso de estudio en el sector salud
}

\author{
Empirical Evaluation of Information Quality: Health Department Case \\ Study
}

\section{Avaliação empírica da qualidade da informação: estudo de caso no setor}

saúde

\author{
José Fidencio López Luna \\ Universidad Politécnica de Victoria, México \\ Universidad Autónoma de Tamaulipas, México \\ jlopezl@upv.edu.mx \\ https://orcid.org/0000-0003-2348-7088
}

Hiram Herrera Rivas

Universidad Politécnica de Victoria, México

hiramhr@gmail.com https://orcid.org/0000-0002-2650-8932

Jorge Arturo Hernández Almazán

Universidad Politécnica de Victoria, México jhernandeza@upv.edu.mx https://orcid.org/0000-0003-1060-6455

\section{Resumen}

Las instituciones suelen emplear sistemas de información a fin de recabar, procesar, almacenar y obtener información de calidad para la toma de decisiones. Contar con información de calidad es un tema clave para las instituciones. La presente investigación pretende percibir problemas concretos de calidad de información en sistemas de información con el fin de proporcionar una mayor calidad de la información en la prestación de servicios de salud pública. Se diseñó un cuestionario con base en un conjunto de dimensiones de calidad de la información. Posteriormente, se aplicó a 160 usuarios finales de sistemas de información de 10 unidades médicas del estado de Tamaulipas, México. Se han obtenido oportunidades específicas de calidad de 


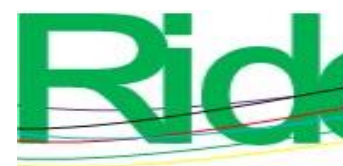

Revista Iberoamericana para la Investigación y el Desarrollo Educativo ISSN 2007 - 7467

información para sistemas de información. Se evidencia la oportunidad de mejora en el tema de calidad de la información en relación con la muestra de sistemas de información considerados en esta investigación. Se sugiere que los resultados obtenidos se consideren en el ámbito académico y profesional a razón de contribuir a elevar la calidad de la información proporcionada por los sistemas de información.

Palabras clave: calidad de la información, sistemas de información, sector salud.

\section{Abstract}

Institutions often use information systems to collect, process, store and obtain quality information for decision-making. Having quality information is a key issue for institutions. This research aims to perceive specific problems of information quality in information systems to help provide a higher quality of the information in the provision of public health services. A questionnaire was designed based on a set of information quality dimensions. Later, it was applied to 160 end users of information systems from 10 medical units in the state of Tamaulipas, Mexico. Specific information quality opportunities have been obtained for information systems. The opportunity for improvement in the issue of information quality is evidenced about to with concerning the sample of information systems considered in this research. It is suggested that the results obtained to be considered in the academic and professional sphere to contribute to raising the quality of the information provided by the information systems.

Keywords: information quality, information systems, health department.

\section{Resumo}

As instituições costumam usar sistemas de informação para coletar, processar, armazenar e obter informações de qualidade para a tomada de decisões. Ter informações de qualidade é uma questão fundamental para as instituições. Esta pesquisa visa perceber problemas específicos de qualidade da informação em sistemas de informação, a fim de proporcionar uma maior qualidade de informação na prestação de serviços públicos de saúde. Um questionário foi elaborado com base em um conjunto de dimensões da qualidade da informação. Posteriormente, foi aplicado a 160 usuários finais de sistemas de informação de 10 unidades médicas no estado de Tamaulipas, México. Oportunidades específicas de qualidade de informação foram obtidas para sistemas de informação. A oportunidade de melhoria na questão da qualidade da informação é evidenciada em relação à amostra de sistemas de informação considerada nesta pesquisa. Sugere-se que 
os resultados obtidos sejam considerados no âmbito acadêmico e profissional, a fim de contribuir para a elevação da qualidade das informações prestadas pelos sistemas de informação.

Palavras-chave: qualidade da informação, sistemas de informação, setor saúde.

Fecha Recepción: Julio 2021

Fecha Aceptación: Enero 2022

\section{Introducción}

Las sociedades posindustriales más desarrolladas conceptualizan la información como conocimiento acumulado y el cimiento del desarrollo económico, político y social (Perez y Lacalle, 2020). Para obtener información competitiva y clave dichas sociedades emplean las tecnologías de la información y la comunicación (TIC) de vanguardia, ya que es importante que esté, la información, actualizada (Guenther, Bat y Mathur, 2020). Dentro del contexto económico de cada país en el área de negocios, los sistemas informáticos cumplen un rol fundamental (Chalupa y Petricek, 2020). Y en esa línea, los administradores de sistemas de información hacen frente a un tema crítico en cuanto a la forma de desarrollar sistemas que brinden grandes beneficios en la calidad de la información para las organizaciones que se crean (Jebraeily, Rahimi, Fazlollahi y Afshar, 2019). Estos sistemas deben proporcionar información oportuna y de calidad en la prestación de servicios (Azeroual, Schöpfel y Ivanovic, 2020) y en la toma de decisiones (Al-Ammary, Al-Doseri, Al-Blushi, Al-Blushi y Aman, 2019). Dada la importancia de los sistemas de información en las sociedades desarrolladas, se ha generado un área del conocimiento para el estudio del correcto desempeño de estos sistemas llamada calidad de la información (Chen, Yu, Hailey y Cui, 2020). Esta área del conocimiento ha suscitado el interés de los investigadores desde hace varios años, aunque especialmente en los más recientes, en distintos contextos (Zhu y Zhou, 2020).

Otra área fundamental de la economía es la salud pública: un país cuyos integrantes no posean buena salud difícilmente podrá tener un buen desarrollo económico. Prueba de ello fue la pandemia de la enfermedad por coronavirus de 2019 (covid-19), gracias a la cual la economía de distintos países se vio afectada (Nájera, 2020). En México, existen problemas de calidad de la información en el sector salud. Particularmente, existe la necesidad de asegurar la medición de calidad de la información en hospitales de tercer nivel (Saturno et al., 2021). Cabe señalar que esta información es reportada a nivel mundial para su análisis y comparación con la de otros países miembros de la Organización para la Cooperación y el Desarrollo Económicos (OCDE). Por lo tanto, es imperativo mejorar los sistemas de información tanto en cantidad y calidad y su 
uso eficaz (Saturno, Martínez, Flores y Poblano, 2019). Para ejemplificar objetivamente la falta de calidad de información pública de la Secretaría de Salud en México durante la pandemia de coronavirus en el 2020, se pueden tomar los datos hospitalarios del sistema Centinela, que mostró casos confirmados, sospechosos y no confirmados de covid-19; dichos datos mostraron una información de dudosa representatividad por ser extrapolados a partir de datos procedentes de una muestra de pocos hospitales (Dyer, 2020).

Ahora bien, a nivel global, la salud pública es un campo de gran volumen de datos que necesita información de alta calidad para la prestación de servicios (Chan y Chang, 2020), para la toma de decisiones y para asegurar la salud de las comunidades (Alolayyan, Alyahya, Alalawin, Shoukat y Nusairat, 2020). La evaluación de la calidad de información es importante para la salud pública, así lo subrayan, por ejemplo, estudios realizados en Brasil (Landmann et al., 2019) y en Kenia (Lucyk, Tang y Quan, 2017). Múltiples funcionalidades clave se han propuesto con base en las TIC en el área de salud para el intercambio de información entre proveedores y sus pacientes (Esmaeilzadeh, Mirzaei y Maddah, 2020), entre las que se encuentran el uso de apoyo clínico y la toma de prescripción electrónica asistida (Choudhary, Agrawal, Dama y Rathod, 2021), así como la capacidad de lograr una mayor participación en la gestión de la población a través de registros electrónicos de enfermedades e informes como instrumentos clínicos (Casillas et al., 2019). Por otro lado, en Estados Unidos existen esfuerzos para crear sistemas de información que sean alimentados por médicos, pacientes, administradores e investigadores para compartir y analizar datos, almacenarlos y que sirvan como una fuente de aprendizaje continuo y sistemático, pues estos sistemas serán una fuente de generación de conocimiento (Bindman, 2019). La creación de estos sistemas se fomenta ya que se sabe actualmente que Internet no proporciona información de salud confiable para personas no profesionales y la calidad de la información de salud en línea requiere de una mejora significativa (Anom, 2020).

Con el fin de lograr una calidad de la información en los sistemas informáticos, se han identificado los componentes esenciales en el proceso de recolección de datos para sistemas de salud pública (Katarahweire, Bainomugisha and Mughal, 2020). Estas dimensiones miden factores tales como la comunicación y actitud hacia los datos, calidad, funcionalidad, soporte tecnológico, liderazgo y financiación. Dichos componentes deberán de ser tomados en cuenta en el desarrollo de sistemas de información de salud pública para lograr una buena calidad de la información. A la fecha, hay una gran cantidad de resultados disponibles en relación con la calidad de la información (Byabazaire, O'Hare y Delaney, 2020), sin embargo, estos resultados han tenido un impacto limitado 


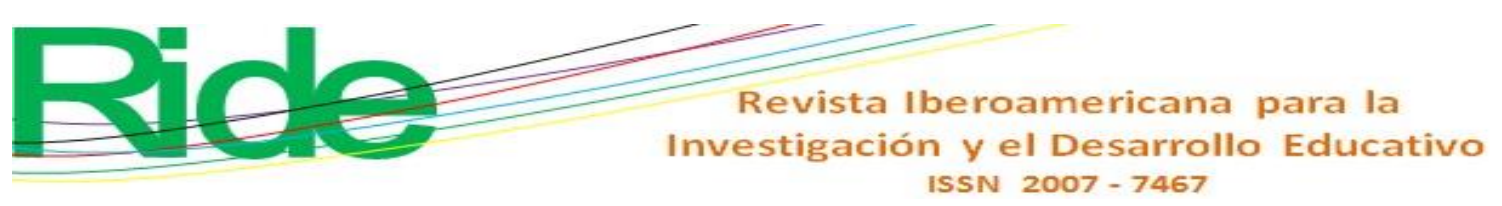

y no se ha logrado combinar y potenciar la teoría y la práctica en su máxima expresión; por otra parte, resulta insuficiente el trabajo realizado en la difusión de los resultados obtenidos respecto al valor que añade la calidad de la información (Bawden y Robinson, 2018). La calidad de la información ha sido considerada como un factor importante que determina el éxito de las aplicaciones orientadas a recoger, almacenar y procesar información (Au, Ho y Chiu, 2021).

Dada la necesidad de sistemas con calidad de la información, se propone un método para detectar oportunidades específicas de calidad de la información en los sistemas informáticos proporcionados por expertos en la materia o manifestados por los usuarios finales. El presente estudio se enfoca en percibir problemas concretos de calidad de información en sistemas informáticos, conceptualizados como oportunidades específicas de mejora para difundirlas en el ámbito académico, a desarrolladores de software y directivos de sistemas, con el fin de ser consideradas en los próximos desarrollos de sistemas de información. Esto ayudará a proporcionar una mayor calidad de la información en la prestación de servicios de salud pública.

\section{Metodología}

\section{Objetivo de investigación}

Detectar las oportunidades comunes de calidad de información en sistemas informáticos seleccionados que pertenecen al sector salud del estado de Tamaulipas.

\section{Diseño de investigación}

Se utilizó un modelo de investigación mixto (cuantitativo y cualitativo). Se recolectaron datos utilizando un instrumento de elaboración propia para detectar las oportunidades comunes de calidad de la información. Dicho instrumento fue un cuestionario creado ad hoc como herramienta para recabar los datos del presente estudio. Para la detección de las áreas de oportunidad en los sistemas de información examinados, se analizó la variable "Nivel de conformidad del usuario final". Además, en cada dimensión de calidad de la información se dejó un espacio para recoger comentarios textuales expresados por el usuario. 


\section{Descripción del cuestionario}

El cuestionario se formó de 35 preguntas cerradas (cuantitativo) y un comentario textual por dimensión (cualitativo) con el propósito de recabar información específica y de facilitar su procesamiento y análisis. Los apartados del cuestionario y la cantidad de preguntas consideradas se muestran en la tabla 1.

Tabla 1. Preguntas del cuestionario agrupado por dimensiones comunes de la calidad de la información (DCCI)

\begin{tabular}{|l|c|}
\hline \multicolumn{1}{|c|}{ DCCI } & $\begin{array}{c}\text { Cantidad de } \\
\text { preguntas }\end{array}$ \\
\hline Disponibilidad & 2 \\
\hline Precisión & 5 \\
\hline Confiabilidad & 3 \\
\hline Completitud & 2 \\
\hline Usabilidad & 2 \\
\hline Facilidad de & 2 \\
\hline manipulación & 4 \\
\hline Objetividad & 2 \\
\hline Relevancia & 2 \\
\hline Seguridad & 2 \\
\hline Oportuna & 2 \\
\hline Valor añadido & 2 \\
\hline Libre de error & 2 \\
\hline Integrada & \\
\hline Ambiente & \\
institucional & \\
\hline
\end{tabular}

Fuente: Elaboración propia

Este instrumento se utilizó debido a que permite recoger datos de muchas personas sin tener que gastar mucho tiempo y dinero (Amorrós, 2019). Las ventajas de utilizar un cuestionario son: bajo costo, fácil de obtener y simplicidad para cuantificar e interpretar información (González y Gamboa, 2020). Además, es una de las técnicas de mayor éxito en el ámbito de las métricas de la calidad de la información debido a que las preguntas están especialmente diseñadas para conocer la percepción de la calidad de la información de los usuarios finales de los sistemas de información. 
En la tabla 2 y 3 se muestran las preguntas asociadas a las DCCI; una parte de las respuestas a estas preguntas fueron cerradas ("Sí", "No", "Lo desconozco"), y también se agregó un cuadro de comentarios textuales para que se anotaran observaciones de los usuarios finales en cada dimensión.

Tabla 2. Cuestionario aplicado a usuarios finales (a)

\begin{tabular}{|c|c|}
\hline DCCI & Pregunta \\
\hline \multirow[t]{2}{*}{ Disponibilidad } & $\begin{array}{l}\text { 1) ¿La información requerida está disponible cuando la } \\
\text { necesita? }\end{array}$ \\
\hline & 2) ¿La información requerida es fácil de obtener? \\
\hline \multirow{5}{*}{ Precisión } & 3) ¿Captura los datos en el momento en que se originan? \\
\hline & 4) ¿Captura los mismos datos en más de un sistema? \\
\hline & $\begin{array}{l}\text { 5) ¿La información proporcionada por el sistema coincide } \\
\text { con la esperada? }\end{array}$ \\
\hline & $\begin{array}{l}\text { 6) ¿La información proporcionada por el sistema contiene } \\
\text { datos innecesarios para la actividad en cuestión? }\end{array}$ \\
\hline & $\begin{array}{l}\text { 7) ¿La información proporcionada por el sistema expresa una } \\
\text { gramática deficiente? }\end{array}$ \\
\hline \multirow{3}{*}{ Confiabilidad } & $\begin{array}{l}\text { 8) ¿La información proporcionada por el sistema procede de } \\
\text { una fuente de confianza? }\end{array}$ \\
\hline & $\begin{array}{l}\text { 9) ¿La información es confiable, sin importar que los datos } \\
\text { sean recolectados de forma manual, mediante sistemas } \\
\text { basados en computadoras o una combinación de estos? }\end{array}$ \\
\hline & $\begin{array}{l}\text { 10) ¿Considera algún margen de error en los datos que } \\
\text { captura? }\end{array}$ \\
\hline \multirow{3}{*}{ Completitud } & 11) ¿Se justifican todos los datos capturados/recolectados? \\
\hline & $\begin{array}{l}\text { 12) ¿Tras la realización de una búsqueda, toda la información } \\
\text { requerida es proporcionada? }\end{array}$ \\
\hline & $\begin{array}{l}\text { 13) ¿La información se proporciona de forma completa y con } \\
\text { el detalle requerido? }\end{array}$ \\
\hline \multirow{3}{*}{ Usabilidad } & 14) ¿La información se proporciona en el formato adecuado? \\
\hline & $\begin{array}{l}\text { 15) ¿La información requerida se proporciona en el medio } \\
\text { adecuado? }\end{array}$ \\
\hline & $\begin{array}{l}\text { 16) ¿El sistema permite manipular la información } \\
\text { proporcionada de acuerdo con lo requerido? }\end{array}$ \\
\hline
\end{tabular}




\begin{tabular}{|c|c|}
\hline $\begin{array}{l}\text { Facilidad de } \\
\text { manipulación }\end{array}$ & $\begin{array}{l}\text { 17) ¿La información proporcionada es aplicable en más de } \\
\text { una actividad? }\end{array}$ \\
\hline \multirow[t]{2}{*}{ Objetividad } & $\begin{array}{l}\text { 18) ¿La información proporcionada posibilita la realización } \\
\text { de actividades concretas? }\end{array}$ \\
\hline & $\begin{array}{l}\text { 19) ¿La información proporcionada por el sistema está } \\
\text { influenciada por sesgos o prejuicios? }\end{array}$ \\
\hline \multirow[t]{4}{*}{ Relevancia } & $\begin{array}{l}\text { 20) ¿Los datos que captura/recaba son útiles para la(s) } \\
\text { actividad(es) en cuestión? }\end{array}$ \\
\hline & $\begin{array}{l}\text { 21) ¿La información proporcionada por el sistema es útil para } \\
\text { la(s) actividad(es) en cuestión? }\end{array}$ \\
\hline & $\begin{array}{l}\text { 22) ¿La información proporcionada sustenta la toma de } \\
\text { decisiones? }\end{array}$ \\
\hline & $\begin{array}{l}\text { 23) ¿La información proporcionada contribuye a incrementar } \\
\text { la calidad de los servicios prestados? }\end{array}$ \\
\hline
\end{tabular}

Fuente: Elaboración propia 
Tabla 3. Cuestionario aplicado a usuarios finales (b)

\begin{tabular}{|c|c|}
\hline DCCI & Pregunta \\
\hline \multirow[t]{2}{*}{ Seguridad } & $\begin{array}{l}\text { 24) ¿Inicia sesión en el sistema para realizar las actividades } \\
\text { asignadas? }\end{array}$ \\
\hline & $\begin{array}{l}\text { 25) ¿Tras haber iniciado sesión en el sistema y transcurrido } \\
\text { cierto tiempo sin utilizarlo, se cierra la sesión } \\
\text { automáticamente? }\end{array}$ \\
\hline \multirow[t]{2}{*}{ Oportuna } & $\begin{array}{l}\text { 26) ¿La información es proporcionada en la periodicidad } \\
\text { requerida? }\end{array}$ \\
\hline & $\begin{array}{l}\text { 27) ¿La información está actualizada a la fecha en que es } \\
\text { requerida? }\end{array}$ \\
\hline \multirow[t]{2}{*}{ Valor añadido } & $\begin{array}{l}\text { 28) ¿La información proporcionada es útil para situaciones } \\
\text { emergentes? }\end{array}$ \\
\hline & 29) ¿La información proporcionada supera las expectativas? \\
\hline \multirow[b]{2}{*}{ Libre de error } & 30) ¿Verifica que los datos que captura/recaba sean correctos? \\
\hline & $\begin{array}{l}\text { 31) ¿La información proporcionada por el sistema presenta } \\
\text { algún tipo de error? }\end{array}$ \\
\hline \multirow[t]{2}{*}{ Integrada } & $\begin{array}{l}\text { 32) ¿Se cuenta con al menos un catálogo de datos } \\
\text { institucional? }\end{array}$ \\
\hline & $\begin{array}{l}\text { 33) ¿Se cuenta con al menos un diccionario de datos } \\
\text { institucional? }\end{array}$ \\
\hline \multirow{2}{*}{$\begin{array}{l}\text { Ambiente } \\
\text { institucional }\end{array}$} & $\begin{array}{l}\text { 34) ¿La organización o relación laboral tiene trascendencia } \\
\text { con la eficacia y credibilidad de los datos? }\end{array}$ \\
\hline & $\begin{array}{l}\text { 35) ¿El ambiente institucional propicia la generación de } \\
\text { información efectiva y creíble? }\end{array}$ \\
\hline
\end{tabular}

Fuente: Elaboración propia

Después de obtener una versión del cuestionario liberada, se procedió a validarlo de dos formas: primero, se realizó un piloto preliminar con el fin de prevenir que existieran errores de ortografía, gramática, palabras o frases ambiguas en la redacción de las preguntas. Segundo, se validó la fiabilidad mediante la evaluación de la consistencia interna a través del cálculo del alfa de Cronbach del cuestionario, pues se sabe que este es una métrica de intercorrelación de consistencia interna entre ítems que conforman la escala (Omary y Kalinga, 2017). 


\section{Análisis de confiabilidad}

Se realizó la prueba de confiabilidad de alfa de Cronbach usando el software estadístico MiniTab versión 16 para validar la consistencia interna de la investigación. De acuerdo con los criterios propuestos por Furr (2021), los valores del alfa para la mayoría de las DCCI son aceptables, a excepción de las dimensiones Precisión, Objetividad, Valor añadido y Libre de error, cuyos valores no son aceptables y, por lo tanto, han sido removidas del estudio, debido a que no hay suficiente coherencia interna en estas dimensiones (ver tabla 4).

Tabla 4. Alfa de Cronbach por DCCI

\begin{tabular}{|l|c|c|}
\hline \multicolumn{1}{|c|}{ DCCI } & $\begin{array}{c}\text { Cantidad de } \\
\text { preguntas }\end{array}$ & Alfa de Cronbach \\
\hline Disponibilidad & 2 & 0.74 \\
\hline Confiabilidad & 3 & 0.58 \\
\hline Completitud & 3 & 0.70 \\
\hline Usabilidad & 2 & 0.77 \\
\hline Facilidad de & 2 & 0.57 \\
manipulación & 4 & 0.72 \\
\hline Relevancia & 2 & 0.65 \\
\hline Seguridad & 2 & 0.67 \\
\hline Oportuna & 2 & 0.64 \\
\hline Integrada & 2 & 0.76 \\
\hline Ambiente institucional & & \\
\hline
\end{tabular}

Fuente: Elaboración propia

\section{Población objetivo}

El universo del estudio fueron 273 usuarios finales, los cuales estaban distribuidos uniformemente en 10 unidades médicas del sector salud del estado de Tamaulipas. Los datos para el cálculo de la muestra representativa, con $95 \%$ de confianza, están en la tabla 5. 
Tabla 5. Tamaño de la muestra

\begin{tabular}{|r|c|c|}
\hline Tamaño de la población & $N$ & 273 \\
\hline Nivel de confianza & $Z$ & 1.96 \\
\hline Proporción esperada & $p$ & 0.5 \\
\hline Complemento & $q$ & 0.5 \\
\hline Margen de error & $E$ & 0.05 \\
\hline Tamaño de la muestra & $n$ & 160 \\
\hline
\end{tabular}

Fuente: Elaboración propia

Las características de la muestra respecto al género, edad y experiencia en uso de la computadora se muestran en la tabla 6. El cuestionario se aplicó a 160 usuarios finales en total, 16 personas por cada unidad médica del sector salud del estado de Tamaulipas, México. Para la aplicación del cuestionario, se invitó a los usuarios finales disponibles a la hora de la visita a las unidades médicas a una plática para explicar los detalles del estudio. Se prestó especial atención en señalar que el cuestionario era anónimo y confidencial, a fin de recabar información con mayor certidumbre. Enseguida, se proporcionó un cuestionario a cada usuario final. Fue importante medir las percepciones reales de los usuarios finales, y la manera de conocerlas fue a través de sus niveles de conformidad con los elementos que midieron la percepción de la calidad de la información. Los datos cuantitativos y cualitativos se recabaron en un mismo momento.

Tabla 6. Características de la muestra

\begin{tabular}{|c|c|c|c|}
\hline \multirow{2}{*}{ Género } & & Frecuencia & Porcentaje \\
\cline { 2 - 4 } & Mombre & 48 & $30 \%$ \\
\hline \multirow{2}{*}{ Edad } & $21-39$ años & 112 & $70 \%$ \\
\cline { 2 - 4 } & $40-60$ años & 80 & $50 \%$ \\
\hline \multirow{2}{*}{$\begin{array}{c}\text { Experiencia en } \\
\text { uso de la } \\
\text { computadora }\end{array}$} & Básico & 36 & $22.5 \%$ \\
\cline { 2 - 4 } & Intermedio & 96 & $60 \%$ \\
\cline { 2 - 4 } & Avanzado & 28 & $17.5 \%$ \\
\hline
\end{tabular}

Fuente: Elaboración propia 
Tabla 7. Valor promedio y desviación estándar por pregunta

\begin{tabular}{|c|c|c|c|c|}
\hline DCCI & $\begin{array}{l}\text { Núm. } \\
\text { Pregunta }\end{array}$ & Media & Moda & $\begin{array}{c}\text { Desviación } \\
\text { estándar }\end{array}$ \\
\hline \multirow{2}{*}{ Disponibilidad } & 1 & 1.24 & 1 & 0.632 \\
\hline & 2 & 1.21 & 1 & 0.589 \\
\hline \multirow{3}{*}{ Confiabilidad } & 8 & 1.18 & 1 & 0.504 \\
\hline & 9 & 1.34 & 1 & 0.667 \\
\hline & 10 & 2.04 & 3 & 0.933 \\
\hline \multirow{3}{*}{ Completitud } & 11 & 1.13 & 1 & 0.454 \\
\hline & 12 & 1.31 & 1 & 0.695 \\
\hline & 13 & 1.36 & 1 & 0.734 \\
\hline \multirow{2}{*}{ Usabilidad } & 14 & 1.11 & 1 & 0.423 \\
\hline & 15 & 1.11 & 1 & 0.385 \\
\hline \multirow{2}{*}{$\begin{array}{l}\text { Facilidad de } \\
\text { manipulación }\end{array}$} & 16 & 1.54 & 1 & 0.859 \\
\hline & 17 & 1.34 & 1 & 0.695 \\
\hline \multirow{4}{*}{ Relevancia } & 20 & 1.04 & 1 & 0.262 \\
\hline & 21 & 1.05 & 1 & 0.273 \\
\hline & 22 & 1.24 & 1 & 0.593 \\
\hline & 23 & 1.17 & 1 & 0.504 \\
\hline \multirow{2}{*}{ Seguridad } & 24 & 1.32 & 1 & 0.709 \\
\hline & 25 & 2.45 & 3 & 0.851 \\
\hline \multirow{2}{*}{ Oportuna } & 26 & 1.24 & 1 & 0.590 \\
\hline & 27 & 1.45 & 1 & 0.804 \\
\hline \multirow{2}{*}{ Integrada } & 32 & 1.66 & 1 & 0.798 \\
\hline & 33 & 2.05 & 2 & 0.775 \\
\hline \multirow{2}{*}{$\begin{array}{c}\text { Ambiente } \\
\text { Institucional }\end{array}$} & 34 & 1.28 & 1 & 0.649 \\
\hline & 35 & 1.33 & 1 & 0.692 \\
\hline
\end{tabular}

Fuente: Elaboración propia

La tabla 8 muestra las DCCI asociadas a cada pregunta del cuestionario; se les asignó un nivel de corrección mayor de mejora cuando el valor de la media de esta pregunta estaba muy cercano a dos, de lo contrario se consideró un nivel de corrección menor. 
Tabla 8. Oportunidades específicas en la calidad de la información para sistemas de información por DCCI

\begin{tabular}{|c|c|c|c|}
\hline DCCI & $\begin{array}{l}\text { Núm. de } \\
\text { pregunta }\end{array}$ & Oportunidad & $\begin{array}{l}\text { Nivel de } \\
\text { mejora }\end{array}$ \\
\hline \multirow{2}{*}{ Disponibilidad } & 1 & $\begin{array}{l}\text { 1) Proporcionar la información requerida } \\
\text { cuando se necesite. }\end{array}$ & Menor \\
\hline & 2 & $\begin{array}{l}\text { 2) Facilitar la información requerida de tal } \\
\text { manera que resulte fácil de obtener. }\end{array}$ & Menor \\
\hline \multirow{3}{*}{ Confiabilidad } & 8 & $\begin{array}{l}\text { 3) Verificar que las fuentes de información } \\
\text { sean confiables. }\end{array}$ & Menor \\
\hline & 9 & $\begin{array}{l}\text { 4) La información que proporcione debe ser } \\
\text { confiable, sin importar que los datos sean } \\
\text { recolectados de forma manual, mediante } \\
\text { sistemas basados en computadoras o una } \\
\text { combinación de estos. }\end{array}$ & Menor \\
\hline & 10 & $\begin{array}{l}\text { 5) Considerar márgenes de error para los datos } \\
\text { que se capturan. }\end{array}$ & Mayor \\
\hline \multirow{3}{*}{ Completitud } & 11 & $\begin{array}{l}\text { 6) Capturar solamente los datos que se } \\
\text { requieran. }\end{array}$ & Menor \\
\hline & 12 & $\begin{array}{l}\text { 7) Proporcionar la información requerida tras } \\
\text { la realización de búsquedas. }\end{array}$ & Menor \\
\hline & 13 & $\begin{array}{l}\text { 8) Proporcionar la información con el detalle } \\
\text { requerido. }\end{array}$ & Menor \\
\hline \multirow{2}{*}{ Usabilidad } & 14 & $\begin{array}{l}\text { 9) Proporcionar la información en el formato } \\
\text { adecuado. }\end{array}$ & Menor \\
\hline & 15 & $\begin{array}{l}\text { 10) Proporcionar la información en el medio } \\
\text { adecuado. }\end{array}$ & Menor \\
\hline \multirow{2}{*}{$\begin{array}{l}\text { Facilidad de } \\
\text { manipulación }\end{array}$} & 16 & $\begin{array}{l}\text { 11) Preparar el sistema de tal manera que } \\
\text { permita a los usuarios finales manejar la } \\
\text { presentación de la información proporcionada } \\
\text { de acuerdo con sus requerimientos. }\end{array}$ & Mayor \\
\hline & 17 & $\begin{array}{l}\text { 12) Adecuar la información que se } \\
\text { proporcione para que sea aplicable a más de } \\
\text { una actividad. }\end{array}$ & Menor \\
\hline
\end{tabular}




\begin{tabular}{|c|c|c|c|}
\hline \multirow{4}{*}{ Relevancia } & 20 & $\begin{array}{l}\text { 13) Verificar que los datos que se capturen } \\
\text { sean verdaderamente útiles para las } \\
\text { actividades en cuestión. }\end{array}$ & Menor \\
\hline & 21 & $\begin{array}{l}\text { 14) Revisar que la información proporcionada } \\
\text { por el sistema sea útil para la(s) actividad(es) } \\
\text { en cuestión. }\end{array}$ & Menor \\
\hline & 22 & $\begin{array}{l}\text { 15) Verificar que la información } \\
\text { proporcionada contribuye a sustentar la toma } \\
\text { de decisiones. }\end{array}$ & Menor \\
\hline & 23 & $\begin{array}{l}\text { 16) Revisar que la información proporcionada } \\
\text { contribuye a incrementar la calidad de los } \\
\text { servicios prestados. }\end{array}$ & Menor \\
\hline \multirow[t]{2}{*}{ Seguridad } & 24 & $\begin{array}{l}\text { 17) Implementar mecanismos de inicio de } \\
\text { sesión en el sistema para poder realizar las } \\
\text { actividades asignadas. }\end{array}$ & Menor \\
\hline & 25 & $\begin{array}{l}\text { 18) Implementar mecanismos efectivos de } \\
\text { cierre de sesión automáticos en los sistemas. }\end{array}$ & Mayor \\
\hline \multirow{2}{*}{ Oportuna } & 26 & $\begin{array}{l}\text { 19) Proporcionar la información en la } \\
\text { periodicidad óptima requerida. }\end{array}$ & Menor \\
\hline & 27 & $\begin{array}{l}\text { 20) Verificar que la información esté } \\
\text { actualizada a la fecha en que sea requerida. }\end{array}$ & Menor \\
\hline \multirow{2}{*}{ Integrada } & 32 & $\begin{array}{l}\text { 21) Fomentar la creación y uso de catálogos } \\
\text { institucionales de datos. }\end{array}$ & Mayor \\
\hline & 33 & $\begin{array}{l}\text { 22) Fomentar la creación y uso de diccionarios } \\
\text { de datos institucionales. }\end{array}$ & Mayor \\
\hline \multirow{2}{*}{$\begin{array}{l}\text { Ambiente } \\
\text { institucional }\end{array}$} & 34 & $\begin{array}{l}\text { 23) Concientizar sobre la relación laboral para } \\
\text { aumentar la eficacia y credibilidad de la } \\
\text { información. }\end{array}$ & Menor \\
\hline & 35 & $\begin{array}{l}\text { 24) Enfatizar en el tema de ambiente } \\
\text { institucional para contribuir en la generación } \\
\text { de información efectiva y creíble. }\end{array}$ & Menor \\
\hline
\end{tabular}

Fuente: Elaboración propia 
Tabla 9. Comentarios textuales de los usuarios finales ordenados de acuerdo con las

DCCI (a)

\begin{tabular}{|c|c|c|}
\hline DCCI & Categoría emergente & Comentario textual \\
\hline \multirow[t]{2}{*}{ Disponibilidad } & Cuando se necesite & $\begin{array}{l}\text { "Solo en el mes actual y no se } \\
\text { pueden sacar reportes de meses } \\
\text { anteriores". } \\
\text { "La información proporcionada por } \\
\text { las diferentes áreas. Se proporciona } \\
\text { incompleta y en tiempo con una } \\
\text { cierta diferencia de retraso a la } \\
\text { fecha". }\end{array}$ \\
\hline & $\begin{array}{l}\text { Cuando resulte fácil de } \\
\text { obtener }\end{array}$ & $\begin{array}{l}\text { "En ocasiones tardamos un poco en } \\
\text { tener la información, pero después } \\
\text { de investigar se consigue". } \\
\text { "Tenemos mucho apoyo por parte } \\
\text { del servicio de Informática". }\end{array}$ \\
\hline \multirow[b]{2}{*}{ Confiabilidad } & $\begin{array}{l}\text { Recolectar o forma de } \\
\text { recolectar }\end{array}$ & $\begin{array}{l}\text { "Un pequeño margen al momento de } \\
\text { capturar manualmente". }\end{array}$ \\
\hline & Margen de error & $\begin{array}{l}\text { "Si bien es de una fuente confiable, } \\
\text { la falta u omisión de datos hace que } \\
\text { no sea tan confiable y esté } \\
\text { incompleta y en nuestro caso el } \\
\text { margen de error casi tiene que ser } \\
\text { cero". } \\
\text { "Se realiza un chequeo de errores } \\
\text { del sistema SAEH antes de enviar la } \\
\text { base de datos a la oficina central". } \\
\text { "Generalmente, el listado de } \\
\text { asistencia mensual contiene } \\
\text { inconsistencias que no lo hace } \\
100 \% \text { confiable". }\end{array}$ \\
\hline
\end{tabular}




\begin{tabular}{|c|c|c|}
\hline \multirow[t]{3}{*}{ Completitud } & Captura de datos requeridos & $\begin{array}{l}\text { "Los sistemas contienen datos que } \\
\text { para un hospital de tercer nivel no } \\
\text { son necesarios y otros que no se } \\
\text { incluyen y requerimos". } \\
\text { "No detalla el tipo de cirugía que se } \\
\text { va a realizar solo la cantidad de } \\
\text { cirugías en SAEH". } \\
\text { "Necesitamos que al sistema se le } \\
\text { integren más opciones para la } \\
\text { búsqueda de la información, ya sea } \\
\text { en fechas o búsqueda de artículo por } \\
\text { proveedor, etc.". }\end{array}$ \\
\hline & $\begin{array}{l}\text { Proporcionar la información } \\
\text { requerida }\end{array}$ & $\begin{array}{l}\text { "La falta de llenado adecuado de los } \\
\text { formatos establecidos hace que la } \\
\text { información que se busca y se } \\
\text { captura sea incompleta". } \\
\text { "No hay acceso a los datos después } \\
\text { de la captura si la información es } \\
\text { insuficiente". }\end{array}$ \\
\hline & $\begin{array}{l}\text { Información con el detalle } \\
\text { requerido }\end{array}$ & $\begin{array}{l}\text { "A veces no arroja información } \\
\text { completa y detallada". }\end{array}$ \\
\hline
\end{tabular}

Fuente: Elaboración propia

Las tablas 9, 10 y 11 muestran el análisis cualitativo asociando categorías emergentes de los datos de acuerdo con los comentarios textuales expresados por los usuarios, estas categorías fueron asociadas a cada dimensión de calidad de la información. Los datos cualitativos fueron contrastados dimensión por dimensión con los resultados obtenidos del análisis cuantitativo para llegar a una conclusión en la oportunidad de mejora, la cual puede ser menor o mayor. En caso de que los datos cualitativos confirmaran el resultado del análisis cuantitativo indicando una oportunidad de corrección mayor, se concluyó en la dimensión correspondiente una oportunidad de mejora mayor. 
Tabla 10. Comentarios textuales de los usuarios finales ordenados de acuerdo con las

DCCI (b)

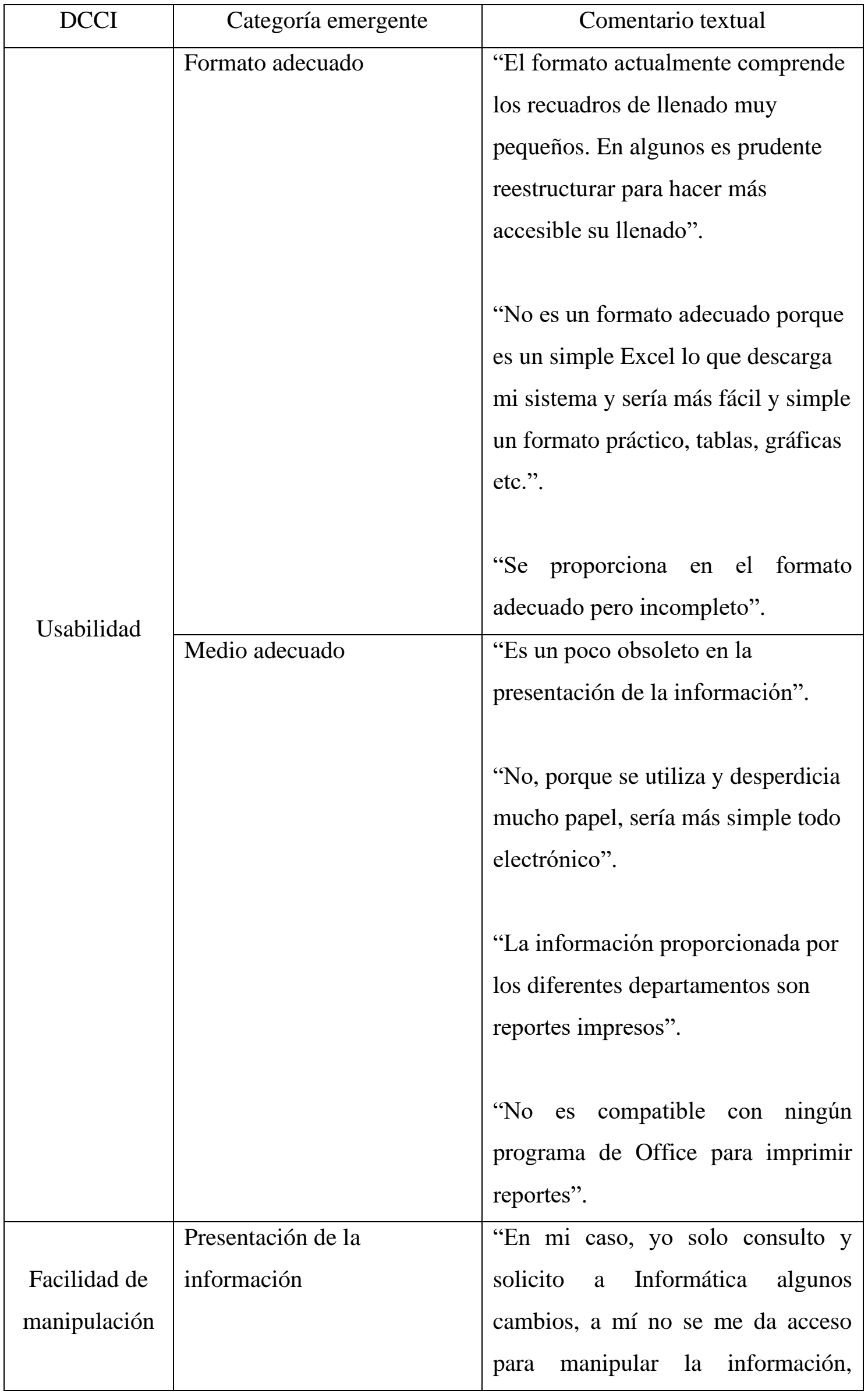




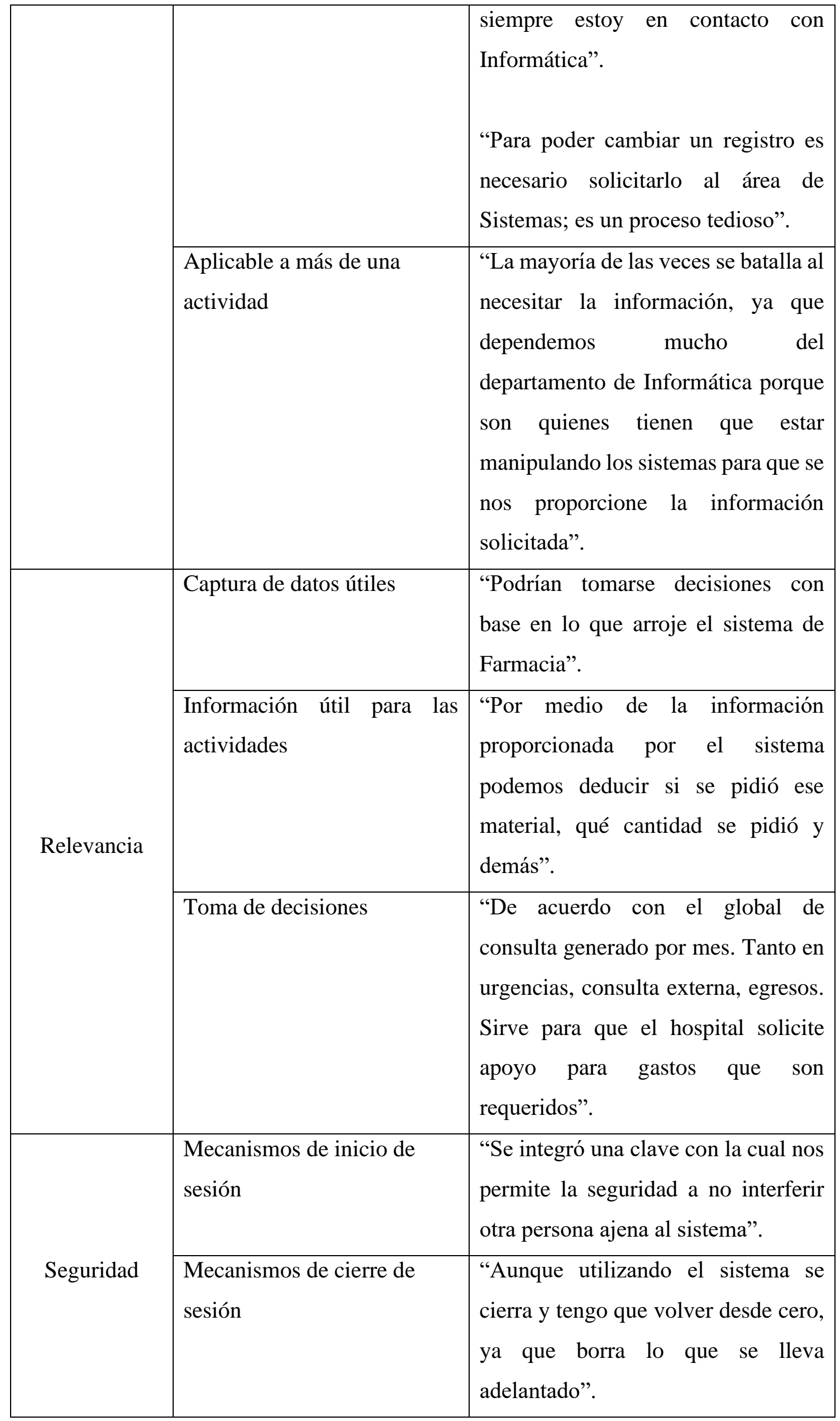


"Se tiene que cerrar manualmente".

Fuente: Elaboración propia

Tabla 11. Comentarios textuales de los usuarios finales ordenados de acuerdo con las DCCI (c)

\begin{tabular}{|c|c|c|}
\hline DCCI & Categoría emergente & Comentario textual \\
\hline \multirow{2}{*}{ Oportuna } & Periodicidad óptima & $\begin{array}{l}\text { "La información es proporcionada } \\
\text { solo si es requerida del año en curso". } \\
\text { "La información es proporcionada en } \\
\text { fecha retrasada, por lo tanto, la } \\
\text { información a enviar se retrasa". } \\
\text { "Algunas veces tenemos retraso para } \\
\text { la entrega de información que se nos } \\
\text { requiere". }\end{array}$ \\
\hline & Información actualizada & $\begin{array}{l}\text { "La falta de apoyo o mala } \\
\text { organización de algunas gentes } \\
\text { provoca que la información no esté } \\
\text { actualizada adecuadamente". } \\
\text { "No está actualizada los días últimos } \\
\text { de cada mes, ya que Contabilidad } \\
\text { revisa el cierre y hasta que termina } \\
\text { nos ponemos a actualizar la } \\
\text { información". }\end{array}$ \\
\hline \multirow[b]{2}{*}{ Integrada } & $\begin{array}{l}\text { Catálogos institucionales de } \\
\text { datos }\end{array}$ & $\begin{array}{l}\text { "Catálogo de especialidades". } \\
\text { “Catálogo de medicamentos". }\end{array}$ \\
\hline & $\begin{array}{l}\text { Diccionarios de datos } \\
\text { institucionales }\end{array}$ & $\begin{array}{l}\text { "Claves Servicio". } \\
\text { "Clasificaciones del Nivel en el } \\
\text { Estudio Socioeconómico" }\end{array}$ \\
\hline
\end{tabular}




\begin{tabular}{|c|c|c|}
\hline & & "Causes 2010". \\
\hline \multirow{3}{*}{$\begin{array}{c}\text { Ambiente } \\
\text { institucional }\end{array}$} & \multirow[t]{3}{*}{ Relación laboral } & $\begin{array}{l}\text { "La falta de apoyo o mala } \\
\text { organización de algunas gentes, la } \\
\text { información no se actualiza } \\
\text { adecuadamente". } \\
\text { "Falta de comunicación entre } \\
\text { enfermera con recepción de admisión } \\
\text { para cambios de cama egresos, por } \\
\text { ejemplo". }\end{array}$ \\
\hline & & $\begin{array}{l}\text { "En algunas ocasiones, el } \\
\text { departamento de Admisión manda al } \\
\text { paciente, le dicen váyase derecho a } \\
\text { archivo clínico y no le especifican a } \\
\text { qué tienen derecho". }\end{array}$ \\
\hline & & $\begin{array}{l}\text { "La información que es requerida de } \\
\text { un paciente hospitalizado, ejemplo, } \\
\text { en Ginecología y se habla a Admisión } \\
\text { hospitalaria para solicitar } \\
\text { información cuando la información se } \\
\text { tiene que dar por recepción de } \\
\text { Ginecología: falta de comunicación". }\end{array}$ \\
\hline
\end{tabular}

Fuente: Elaboración propia

\section{Discusión}

A través del análisis de los resultados expresados por los usuarios finales, se observaron oportunidades específicas de calidad de la información para sistemas informáticos. A continuación, se describen los hallazgos encontrados:

- Disponibilidad: la percepción de los usuarios finales indicó que los sistemas informáticos son eficientes en cuanto al acceso a la información cuando se necesita y que dicha información les resulta fácil de obtener, sin embargo, esto no sucede en su totalidad, ya que algunos usuarios finales manifestaron que para algunos meses del año en los sistemas no se puede obtener la información. Finalmente, se determina una menor oportunidad de mejora en esta dimensión. 


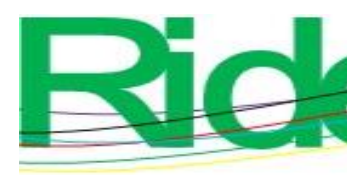

Revista Iberoamericana para la Investigación y el Desarrollo Educativo ISSN $2007-7467$

- $\quad$ Confiabilidad: se encontró que hay certeza entre los usuarios finales para las fuentes de información y la recolección de los datos, sin embargo, algunos usuarios manifiestan que hay margen de error en la captura de los datos. Además, señalan que los datos son verificados en el proceso de captura, y que la información sigue siendo confiable a pesar de la existencia de omisiones en la captura de algunos datos. Por consecuencia, existe una oportunidad de mejora mayor en la captura de los datos en cuanto al margen de error de estos.

- $\quad$ Completitud: los hallazgos mostraron que para los sistemas informáticos los usuarios finales mayormente capturan los datos requeridos, el sistema les proporciona la información que requieren con el detalle indicado. Lo anteriormente expuesto contrasta con la opinión de algunos usuarios finales, quienes señalan que para algunos casos se capturan más datos de los requeridos y que el sistema les proporciona información incompleta. Los datos cuantitativos y cualitativos difieren, por lo tanto, no se puede determinar si existe oportunidad de mejora en la dimensión Completitud.

- Usabilidad: a decir de los usuarios finales, los sistemas informáticos les proporcionan los datos en el formato y medio adecuado cuando así lo requieren. No obstante, se señala que entre departamentos la información básicamente se proporciona impresa, cuando es requerida en forma digital. Los datos cuantitativos y cualitativos difieren entre sí, por lo tanto, no se puede determinar si existe oportunidad de mejora en la dimensión.

- $\quad$ Facilidad de manipulación: la información proporcionada a los usuarios no se presentó como se requirió; por otro lado, la información proporcionada fue aplicable a más de una actividad. En este sentido, algunos usuarios finales manifestaron descontento por la imposibilidad de poder actualizar datos después de haber transcurrido un tiempo considerable de la captura de estos. Por lo anterior, existe una oportunidad de mejora mayor en esta dimensión para presentar la información como la requieren algunos usuarios.

- Relevancia: en esta dimensión se encontró que la apreciación de los usuarios finales a los datos capturados es realmente útil, así como la información proporcionada, mientras que la información proporcionada les contribuye a sustentar la toma de decisiones, así como a incrementar la calidad de los servicios prestados. Lo anterior confirma una oportunidad de mejora menor en la dimensión.

- Seguridad: los hallazgos mostraron que los sistemas informáticos cuentan con mecanismos de inicio de sesión efectivos, a decir de algunos usuarios finales, mientras que hacen falta implementar mecanismos de cierre de sesión automáticos. Por 


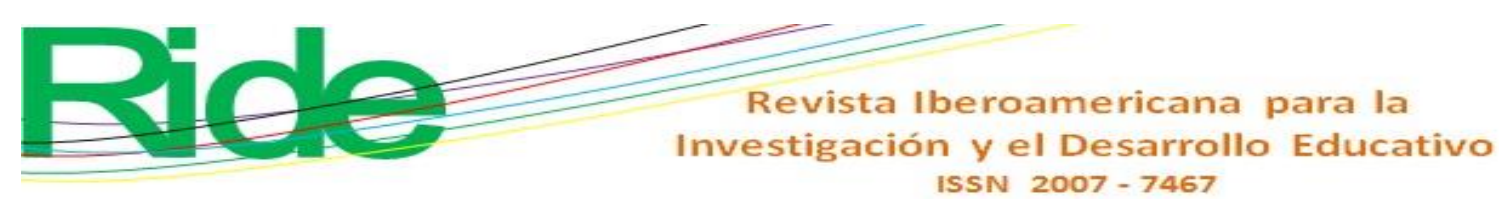

consecuencia, existe una oportunidad de mejora mayor en la dimensión para implementar mecanismos de cierre de sesión automáticos.

- Oportuna: se encontró que la información se proporciona con la periodicidad óptima y que está actualizada a la fecha que es requerida. Sin embargo, a decir de algunos usuarios, los sistemas proporcionan solo la información del año en curso y no está actualizada al cierre de cada mes, generalmente por la falta de coordinación entre el personal de la institución. Los datos cuantitativos y cualitativos difieren entre sí, por lo tanto, no se puede determinar si existe oportunidad de mejora en la dimensión oportuna.

- Integrada: esta dimensión mostró que la percepción de los usuarios finales apunta a que se requieren implementar catálogos y diccionarios de datos institucionales en los sistemas informáticos. Lo anterior denota una oportunidad de mejora mayor en esta dimensión.

- $\quad$ Ambiente institucional: se encontró que los usuarios finales manifiestan estar conscientes del hecho de que la relación laboral y el ambiente institucional es importante en la eficacia y credibilidad de la información. Sin embargo, algunos usuarios finales manifiestan que hace falta una mayor organización para el manejo de la información entre departamentos. Se concluye que existe una oportunidad de mejora mínima en esta dimensión.

Los interesados en continuar esta investigación podrían dirigir sus trabajos a la formación de capital humano en área de calidad de información y el impacto que tendría en el desarrollo de software, la evaluación de la cultura de datos compartidos, abordar esquemas de integración de sistemas de información, estudiar metodologías para la integración e interoperabilidad entre sistemas informáticos, lo que no es una tarea fácil. Lo anterior con la premisa de equilibrar la balanza entre el conocimiento y tecnologías generados, por una parte, y por la otra, la práctica, es decir, lograr gestionar y transferir el conocimiento y la tecnología producidos a las sociedades que lo ameritan. De acuerdo con Bawden y Robinson (2018), esta filosofía en materia de calidad de la información generalmente no se lleva a cabo. Finalmente, mencionaremos que nuestro estudio estuvo limitado a instituciones del sector salud del estado de Tamaulipas. 


\section{Referencias}

Al-Ammary, J. H., Al-Doseri, S., Al-Blushi, Z., Al-Blushi, N. and Aman, M. (2019). Strategic information systems planning in Kingdom of Bahrain: Factors and impact of adoption. International Journal of Business Information Systems, 30(4), $387-410$.

Alolayyan, M. N., Alyahya, M. S., Alalawin, A. H., Shoukat, A. and Nusairat, F. T. (2020). Health information technology and hospital performance the role of health information quality in teaching hospitals. Heliyon, 6(10). Retrieved from https://doi.org/10.1016/j.heliyon.2020.e05040.

Amorrós, L. (2019). Actitudes y conocimientos de entornos digitales. Cuestionario ACMI para contextos socioeducativos. Madrid, España: Dykinson.

Anom, B. Y. (2020). Ethics of Big Data and artificial intelligence in medicine. Ethics, Medicine and Public Health, 15. Retrieved from https://doi.org/10.1016/j.jemep.2020.100568.

Au, C. H., Ho, K. K. and Chiu, D. K. (2021). Stopping healthcare misinformation: The effect of financial incentives and legislation. Health Policy, 125(5), 627-633.

Azeroual, O., Schöpfel, J. and Ivanovic, D. (2020). Influence of Information Quality via Implemented German RCD Standard in Research Information Systems. Data, 5(2). Retrieved from https://doi.org/10.3390/data5020030.

Bawden, D. and Robinson, L. (2018). Curating the infosphere: Luciano Floridi's Philosophy of Information as the foundation for Library and Information Science. Journal of Documentation, 74(1), 2-17. Retrieved from https://doi.org/https://doi.org/10.1108/JD-07-2017-0096.

Bindman, A. B. (2019). Learning healthcare systems: a perspective from the US. Public Health Resolution and Practice, 29(3). Retrieved from https://doi.org/10.17061/phrp2931920.

Byabazaire, J., O’Hare, G. and Delaney, D. (2020). Data Quality and Trust: Review of Challenges and Opportunities for Data Sharing in IoT. Electronics, 9(12). Retrieved from https://doi.org/10.3390/electronics9122083.

Casillas, A., Perez, G., Abhat, A., Gutierrez, G., Olmos, T. T., Mendez, C., Mahajan, A., Brown, A. and Moreno, G. (2019). Su salud a la mano (your health at hand): patient perceptions about a bilingual patient portal in the Los Angeles safety net. Journal of the American Medical Informatics Association, 26(12), 1525-1535. 


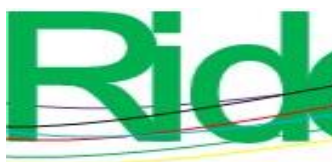

Revista Iberoamericana para la Investigación y el Desarrollo Educativo ISSN $2007-7467$

Chalupa, S. and Petricek, M. (2020). Using Technology and Customer Behavior Characteristics to Improve Hotel Sales Performance. TEM Journal, 9(2), 573-577. Retrieved from https://doi.org/10.18421/tem92-20.

Chan, C. L. and Chang, C. C. (2020). Big Data, Decision Models, and Public Health. International Journal Environmental Research and Public Health, 17(18). Retrieved from https://doi.org/10.3390/ijerph17186723.

Chen, H., Yu, P., Hailey, D. and Cui, T. (2020). Identification of the essential components of quality in the data collection process for public health information systems. Health Informatics Journal, 26(1), 664-682.

Choudhary, K., Agrawal, T., Dama, R. and Rathod, M. (2021). Voice Based EPrescription. SSRN Electronic Journal. Retrieved from https://doi.org/10.2139/ssrn.3867317.

Dyer, O. (2020). Covid-19: Mexico acknowledges 50000 more deaths than official figures show. The BMJ, 371. Retrieved from https://doi.org/10.1136/bmj.m4182.

Esmaeilzadeh, P., Mirzaei, T. and Maddah, M. (2020). The effects of data entry structure on patients' perceptions of information quality in Health Information Exchange (HIE). International Journal of Medical Informatics, 135. Retrieved from https://doi.org/10.1016/j.ijmedinf.2019.104058.

Furr, R. M. (2021). Psychometrics: An Introduction. Thousand Oaks, United States: SAGE publications.

Guenther, J., Bat, M. and Mathur, D. (2020). Knowledge intersections: red dirt knowledge from the heart Rural Society, 29(3), 151-153. Retrieved from https://doi.org/10.1080/10371656.2020.1842596.

González, M. y Gamboa, M. E. (2020). Sistema de acciones para captar información en Oficina Nacional de Estadística e Información del municipio Manatí. Didasc@lia: Didáctica y Educación, 11(2), 168-192.

Jebraeily, M., Rahimi, B., Fazlollahi, Z. Z. and Afshar, H. L. (2019). Using SERVQUAL model to assess hospital information system service quality. Hormozgan Medical Journal, 23(1).

Katarahweire, M., Bainomugisha, E. and Mughal, K. A. (2020). Data Classification for Secure Mobile Health Data Collection Systems. Development Engineering, 5. Retrieved from https://doi.org/10.1016/j.deveng.2020.100054.

Landmann, C., Leal, M., Esteves, A. P., da Silva, W, Germano, P., Nogueira, G., Borges, P., Martins, N. and Honorato, P. (2019). Evaluation of data from the Brazilian 


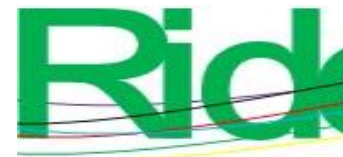

Revista Iberoamericana para la Investigación y el Desarrollo Educativo ISSN 2007 - 7467

Information System on Live Births (SINASC). Cadernos de Saúde Pública, 35(10). Retrieved from https://doi.org/10.1590/0102-311X00214918.

Lucyk, K., Tang, K. and Quan, H. (2017). Barriers to data quality resulting from the process of coding health information to administrative data: a qualitative study. Health Services Research, 17(1), 1-10. Retrieved from https://doi.org/10.1186/s12913-017-2697-y.

Nájera, H. (2020). Desigualdades institucionales de salud en México frente al covid-19. En Cordera, R. y Provencio, E. (coords.), Cambiar el rumbo: el desarrollo tras la pandemia (pp. 102-110). Ciudad de México, México: Universidad Autónoma de México.

Omary, Z. D. and Kalinga, E. (2017). Assessing Users Satisfaction with Tanzanians Public Health Supply Chain Electronic Logistic Management Information System. Journal of Health Informatics in Developing Countries, 11(2).

Perez, M. and Lacalle, M. (2020). The impact of knowledge diffusion on economic growth across countries. World Development, 132. Retrieved from https://doi.org/10.1016/j.worlddev.2020.104995.

Saturno, P. J., Martínez, I., Flores, S. y Poblano, O. (2019). Calidad del sistema de información en salud: análisis comparativo de indicadores reportados, México OCDE 2010-2016. Salud Pública de México, 61(2), 184-192. Recuperado de https://doi.org/https://doi.org/10.21149/9688.

Saturno, P. J., Poblano, O., Flores, S., Martínez, I., Vieyra, W. y Halley, M. E. (2021). Carencias y variabilidad en la calidad de la atención a neonatos hospitalizados en México. Estudio transversal en 28 hospitales públicos. Salud Pública de México, 63(2), 180-189. Recuperado de https://doi.org/10.21149/11616.

Zhu, Y. and Zhou, Z. (2020). Research on Power Demand Side Information Quality Indicators and Evaluation Based on Grounded Theory Approach. Information, 11(10). Retrieved from https://doi.org/10.3390/info11100477. 


\begin{tabular}{|c|c|}
\hline Rol de Contribución & Autor (es) \\
\hline Conceptualización & José Fidencio López Luna \\
\hline Metodología & Hiram Herrera Rivas \\
\hline Software & Jorge Arturo Hernández Almazán \\
\hline Validación & José Fidencio López Luna \\
\hline Análisis Formal & Hiram Herrera Rivas \\
\hline Investigación & José Fidencio López Luna \\
\hline Recursos & José Fidencio López Luna \\
\hline Curación de datos & Jorge Arturo Hernández Almazán \\
\hline $\begin{array}{l}\text { Escritura - Preparación del } \\
\text { borrador original }\end{array}$ & Hiram Herrera Rivas \\
\hline Escritura - Revisión y edición & Hiram Herrera Rivas \\
\hline Visualización & Jorge Arturo Hernández Almazán \\
\hline Supervisión & José Fidencio López Luna \\
\hline Administración de Proyectos & Jorge Arturo Hernández Almazán \\
\hline Adquisición de fondos & José Fidencio López Luna \\
\hline
\end{tabular}

